\title{
Differences in Radiation Exposure of CT-Guided Percutaneous Manual and Powered Drill Bone Biopsy
}

\author{
Sebastian Zensen ${ }^{1}\left(\mathbb{D} \cdot\right.$ Sumitha Selvaretnam $^{1} \cdot$ Marcel Opitz $^{1} \cdot$ Denise Bos $^{1} \cdot$ \\ Johannes Haubold $^{1} \cdot$ Jens Theysohn $^{1} \cdot$ Michael Forsting $^{1} \cdot$ Nika Guberina $^{2}$. \\ Axel Wetter ${ }^{1,3}$
}

Received: 10 November 2020/Accepted: 16 April 2021/Published online: 11 May 2021

(C) The Author(s) 2021

\begin{abstract}
Purpose Apart from the commonly applied manual needle biopsy, CT-guided percutaneous biopsies of bone lesions can be performed with battery-powered drill biopsy systems. Due to assumably different radiation doses and procedural durations, the aim of this study is to examine radiation exposure and establish local diagnostic reference levels (DRLs) of CT-guided bone biopsies of different anatomical regions.

Methods In this retrospective study, dose data of 187 patients who underwent CT-guided bone biopsy with a manual or powered drill biopsy system performed at one of three different multi-slice CT were analyzed. Between January 2012 and November 2019, a total of 27 femur (A), 74 ilium (B), 27 sacrum (C), 28 thoracic vertebrae (D) and 31 lumbar vertebrae (E) biopsies were included. Radiation exposure was reported for volume-weighted CT dose index $\left(\mathrm{CTDI}_{\mathrm{vol}}\right)$ and dose-length product (DLP).

Results $\mathrm{CTDI}_{\mathrm{vol}}$ and DLP of manual versus powered drill biopsy were (median, IQR): A: 56.9(41.4-128.5)/66.7 (37.6-76.2)mGy, 410(203-683)/303(128-403)mGy·cm, B: 83.5(62.1-128.5)/59.4(46.2-79.8)mGy, 489(322-472)/ 400(329-695)mGy·cm, C: 97.5(71.6-149.2)/63.1(49.1-
\end{abstract}

Sebastian Zensen

sebastian.zensen@uk-essen.de

1 Department of Diagnostic and Interventional Radiology and Neuroradiology, University Hospital Essen, Hufelandstraße 55, 45147 Essen, Germany

2 Department of Radiotherapy, University Hospital Essen, Hufelandstraße 55, 45147 Essen, Germany

3 Department of Diagnostic and Interventional Radiology, Neuroradiology, Asklepios Klinikum Harburg, Eißendorfer Pferdeweg 52, 21075 Hamburg, Germany
83.7)mGy, 627(496-740)/404(316-515)mGy·cm, D: 67.0 (40.3-86.6)/39.7(29.9-89.0)mGy, 392(267-596)/207(166402) $\mathrm{mGy} \cdot \mathrm{cm}$ and E: $100.1(66.5-162.6) / 62.5(48.0-90.0)$ mGy, 521(385-619)/315(240-452)mGy·cm. Radiation exposure with powered drill was significantly lower for ilium and sacrum, while procedural duration was not increased for any anatomical location. Local DRLs could be depicted as follows $\left(\mathrm{CTDI}_{\mathrm{vol}} / \mathrm{DLP}\right): \mathbf{A}: 91 \mathrm{mGy} / 522$ $\mathrm{mGy} \cdot \mathrm{cm}, \mathbf{B}: 90 \mathrm{mGy} / 530 \mathrm{mGy} \cdot \mathrm{cm}, \mathbf{C}: 116 \mathrm{mGy} / 740$ $\mathrm{mGy} \cdot \mathrm{cm}, \mathbf{D}: 87 \mathrm{mGy} / 578 \mathrm{mGy} \cdot \mathrm{cm}$ and E: $115 \mathrm{mGy} / 546$ $\mathrm{mGy} \cdot \mathrm{cm}$. The diagnostic yield was $82.4 \%$ for manual and $89.4 \%$ for powered drill biopsies.

Conclusion Use of powered drill bone biopsy systems for CT-guided percutaneous bone biopsies can significantly reduce the radiation burden compared to manual biopsy for specific anatomical locations such as ilium and sacrum and does not increase radiation dose or procedural duration for any of the investigated locations.

Level of Evidence Level 3.

Keywords computed tomography · bone biopsy · radiation exposure $\cdot$ diagnostic reference level

\section{Introduction}

CT-guided percutaneous bone biopsies play a key role for the diagnostic work-up of skeletal lesions such as inflammatory and malignant processes. Compared with standard open biopsy, CT-guided approaches are less invasive and provide a sufficient specimen yield [1-3]. Furthermore, CT-guided biopsies have a lower complication rate and are 
generally well tolerated $[4,5]$. These procedures can be performed either with a manual approach by placing a bone needle within the skeletal lesion, possibly achieved by hammering technique to perforate the cortical bone, or with a powered drill bone biopsy system such as the commercially available Arrow ${ }^{\circledR}$ OnControl ${ }^{\circledR}$ powered bone access system. For this battery-powered drill, decreased procedural duration, improved user-friendliness and lower pain perception were reported $[6,7]$. Furthermore, the powered drill approach provides a higher diagnostic yield for sclerotic lesions [4]. Alongside these benefits of manual and powered drill CT-guided bone biopsies, CT entails a radiation burden and is a high-dose imaging technique, which causes the major part of collective effective dose of all medical imaging [8,9]. While some recent studies reported radiation doses of CT-guided bone biopsies $[4,10-12]$, further detailed dose assessment and comparison between different anatomical regions are needed to optimize radiation protection. Additionally, specific reports of diagnostic reference levels (DRL) are rare [13]. For various indications, DRLs were established to limit radiation exposure of radiological imaging modalities [14]. To compare and evaluate local radiation exposure distributions and optimize radiation protection, $75^{\text {th }}$ percentiles of dose metric distributions are often used as DRL [15].

The aim of this study was to evaluate the radiation exposure and procedural duration of CT-guided percutaneous manual and powered drill bone biopsies and to establish local DRLs.

\section{Materials and Methods}

\section{Patient Cohort}

Between January 2012 and November 2019, dose data of all CT-guided percutaneous bone biopsies at our center were included, which provided full information for dose metrics, precisely reported anatomical location as well as technical and procedural duration information. Patients were identified using the radiological information system (RIS). Following anatomical locations were included: femur, ilium, sacrum, thoracic and lumbar vertebrae. Clinical information was extracted from the report archived in the RIS. Ethical approval for this retrospective singlecenter study was granted by the institutional review board and the requirement to obtain informed consent was waived (19-8579-BO).

\section{CT Scanners and Biopsy Equipment}

All interventions were performed by experienced interventional radiologists at one of three commercially available, modern multi-slice CT scanners: single-source 128-slice SOMATOM Definition AS +, dual-source 128-slice SOMATOM Definition Flash and dual-source 192-slice SOMATOM Force (all: Siemens Healthineers, Erlangen, Germany). At dual-source CT scanners, only one tube was used. For all scans, the tube voltage was $120 \mathrm{kV}$ and the rotation time $0.5 \mathrm{~s}$. Further technical settings according to CT scanner are shown in Table 1. For manual bone biopsy, commercially available 10-, 11- or 13-gauge bone biopsy needles such as the Ostycut ${ }^{\circledR}$ bone biopsy needle (Bard, Covington, USA) and other bone biopsy sets (Stryker, Kalamazoo, USA) were used. For battery-powered drill bone biopsy, the Arrow ${ }^{\circledR}$ On Control ${ }^{\circledR}$ powered bone access system with attachable 11-gauge biopsy needle (Teleflex, Wayne, USA) was applied, which is a handheld powered drill with electric drive and manual guidance of the drilling channel (Fig. 1).

\section{Definitions}

To determine the diagnostic yield of the CT-guided bone biopsies, the pathology reports were checked to see if a diagnosis could be made from the specimen. A biopsy was considered diagnostic if the specimen was eligible for histological evaluation.

To investigate the dose difference between bone biopsies of osteolytic and osteoblastic lesions, the average density of the lesion was determined. An average density below $250 \mathrm{HU}$ was considered osteolytic, above that osteoblastic.

Bone biopsies were divided into superficial and deep biopsies according to the depth of the lesion, which was measured from the skin puncture site to the site of the tip of the biopsy needle or drill bit within the bone lesion. A depth up to $70 \mathrm{~mm}$ was considered superficial, above that depth was considered deep.

The assessed procedural duration refers to the period between the start of the intra-procedural, i.e., biopsyguiding sequence with acquisition of the first scan after puncture to its end with successful placement of the biopsy needle in the lesion but before acquisition of the postbiopsy scan for documentation and recording of possible complications.

\section{Bone Biopsy Procedure}

First, a prebioptic scan was obtained for biopsy planning. Subsequently, the area of the planned puncture site was locally anesthetized. The manual system contains a disposable biopsy cannula with internal stylet. Intraosseously, the inner stylet was removed and the biopsy needle advanced through the lesion to the desired depth using rotary motion or hammering technique using a mallet. 
Table 1 Technical parameters of CT-guided percutaneous bone biopsy at three different multi-slice Siemens CT scanners

\begin{tabular}{|c|c|c|c|c|c|c|c|c|c|c|c|c|c|c|c|}
\hline \multirow[t]{2}{*}{ CT scanner* } & \multicolumn{3}{|l|}{ Femur } & \multicolumn{3}{|l|}{ Ilium } & \multicolumn{3}{|c|}{ Sacrum } & \multicolumn{3}{|c|}{ Thoracic vertebrae } & \multicolumn{3}{|c|}{ Lumbar vertebrae } \\
\hline & $\mathrm{AS}+$ & Flash & Force & $\mathrm{AS}+$ & Flash & Force & $\mathrm{AS}+$ & Flash & Force & $\mathrm{AS}+$ & Flash & Force & $\mathrm{AS}+$ & Flash & Force \\
\hline$n$ & 14 & 11 & 2 & 16 & 45 & 13 & 4 & 17 & 6 & 11 & 15 & 2 & 8 & 18 & 5 \\
\hline $\begin{array}{l}\text { Tube current- } \\
\text { time product } \\
\text { (mAs) }\end{array}$ & 50 & 50 & 150 & 50 & 50 & 150 & 50 & 50 & 150 & 50 & 50 & 165 & 50 & 50 & 150 \\
\hline $\begin{array}{l}\text { Slice thickness } \\
(\mathrm{mm})\end{array}$ & 1.2 & 1.2 & 5.0 & 1.2 & 1.2 & 5.0 & 1.2 & 1.2 & 5.0 & 1.2 & 1.2 & $\begin{array}{l}1.0 / \\
5.0\end{array}$ & 1.2 & 1.2 & 2.5 \\
\hline
\end{tabular}

${ }^{*}$ AS + : SOMATOM Definition AS + ; Flash: SOMATOM Definition Flash; Force: SOMATOM Force (all: Siemens Healthineers, Erlangen, Germany)

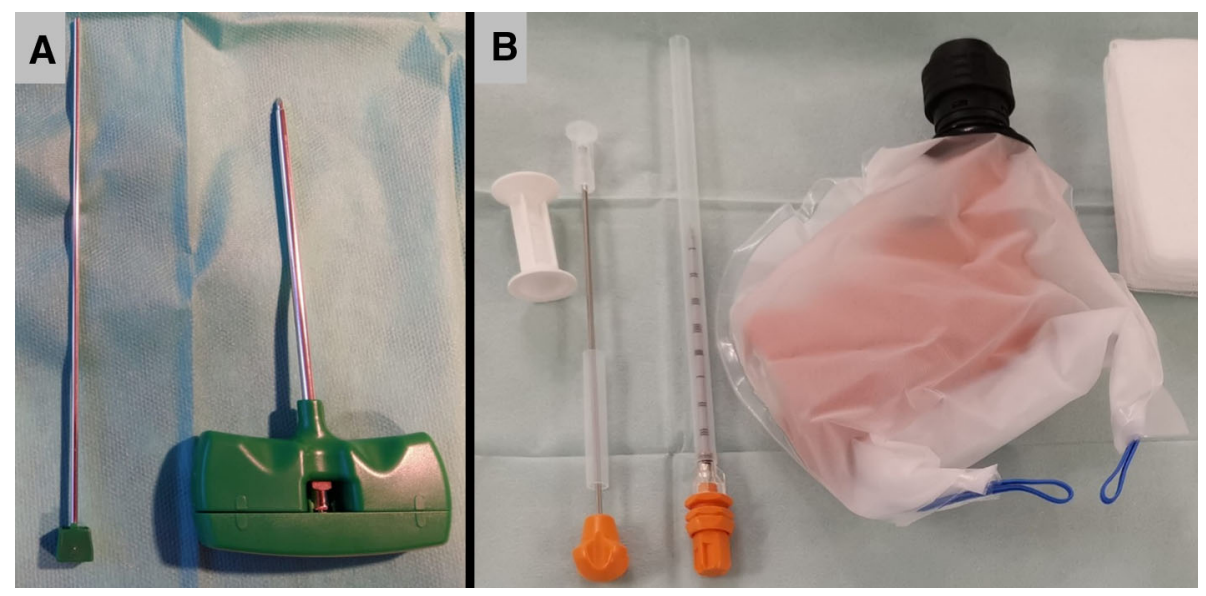

Fig. 1 Manual and powered drill bone biopsy systems. Shown in $\mathbf{A}$ is a typical commercially available manual bone biopsy kit consisting of the biopsy needle (right) and an obturator (left) for pushing the specimen out of the needle. Shown in $\mathbf{B}$ is the reusable, non-sterile battery-powered drill (Arrow OnControl, Teleflex, Wayne, USA)

Subsequently, the system was completely removed and the specimen was carefully extruded from the needle using an obturator. The powered drill system includes a handheld reusable electric drill with a sealed lithium-ion battery to which a disposable bone biopsy needle is attached. The drill does not have a hammer function and the attachable 11 -gauge biopsy needle is available in a length of 4 or six inches (102 $\mathrm{mm}$ or $152 \mathrm{~mm}$ ) and is coaxial in design with an outer cannula and an inner stylet with a beveled tip [12]. The non-sterile drill was wrapped in a sterile bag prior to biopsy and connected to the biopsy needle via a connector. Once the needle tip was placed immediately in front of the bone lesion, the drill was removed from the connector and the inner stylet was removed from the biopsy needle. The drill was then reconnected and the biopsy needle was used to drill through the bone lesion to the desired depth. The system was then completely removed and the specimen in the biopsy needle was carefully pushed out using the stylet. In both approaches, intermittent biopsy-guiding CT scans (right), which was wrapped in a sterile bag prior to biopsy, and an associated disposable 11-gauge 4 inch $(102 \mathrm{~mm})$ biopsy needle (center) and an obturator (left) for pushing the specimen out of the needle

were taken for positional control. As soon as the biopsy needle could be delineated intralesionally, the internal stylet was removed and the tissue sample was collected. The biopsy system was then removed and a post-bioptic scan was performed for documentation and to exclude complications.

\section{Dose Assessment}

For dose assessment, examination data and dose measurements were extracted from the Digital Imaging and Communications in Medicine (DICOM) header and from the Radiation Dose Structured Report stored in the Picture Archiving and Communication System (PACS). Dose assessments referred to the $32 \mathrm{~cm}$ diameter standard polymethyl methacrylate (PMMA) CT dosimetry phantom. Assessed radiation exposure indices were the volumeweighted CT dose index $\left(\mathrm{CTDI}_{\mathrm{vol}}\right)$ and dose-length product (DLP). Although they do not directly represent the 
dose to an individual patient, $\mathrm{CTDI}_{\mathrm{vol}}$ and DLP quantify the radiation dose output of a CT scanner and may help to ensure lower radiation exposures. DRLs were set at the $75^{\text {th }}$ percentile of dose distribution. Both the manual and powered drill bone biopsies were performed under CT guidance in step-and-shoot technique. For both approaches, multiple CT scans were needed to monitor the location of the biopsy needle, respectively, the drill tip during the biopsy-guiding scans. All DLP values of the biopsy-guiding scans were added to a total DLP, so that all scans necessary for the biopsy and possibly acquired CT spirals were included in the total DLP. To emphasize the differences of radiation dose contributed to the application of manual versus powered drill bone biopsy system, radiation doses of biopsy-guiding scans were analyzed additionally with exclusion of pre- and post-bioptic scans. Dose assessment was also performed for different subgroups in relation to characteristics of the bone lesions, that is, in terms of density, depth, anatomical location, suspected etiology, and technical parameters such as needle diameter and protocols on CT scanners.

\section{Statistics and Data Analysis}

Descriptive statistics were performed using GraphPad Prism 5.01 (GraphPad Software, San Diego, USA). To determine normal distribution Kolmogorov-Smirnov, Shapiro-Wilk and D'Agostino-Pearson test were applied. Normally distributed data are reported as mean \pm standard deviation (SD), non-normally distributed data as median and interquartile range (IQR). Mann-Whitney U test was applied to compare radiation indices between manual and powered drill approaches. Kruskal-Wallis test with DunnBonferroni post hoc test was performed to compare procedural durations and DLP values of manual biopsies with different needle diameters. A p value lower than 0.05 was considered statistically significant.

\section{Results}

\section{Patient Cohort}

In our retrospective study, 187 patients who underwent a CT-guided percutaneous bone biopsy between January 2012 and November 2019 could be included for evaluation. Median age was 57.4 years (IQR 42.9-67.6, total range 7.9-86.5 years). Median BMI of patients with manual biopsy was $25.0 \mathrm{~kg} / \mathrm{m}^{2}$ (IQR 22.8-28.0) and of patients with powered drill biopsy $24.2 \mathrm{~kg} / \mathrm{m}^{2}$ (IQR 22.1-27.1). Statistical analysis showed that there was no significant difference in BMI $(p=0.3065)$. Included datasets comprised a total (manual/powered drill) of 27 (13/14) femur
(A), 74 (27/47) ilium (B), 27 (11/16) sacrum (C), 28 (13/ 15) thoracic vertebrae (D) and 31 (10/21) lumbar vertebrae (E) biopsies. Regarding diagnostic yield, $82.4 \%$ (61 out of 74) manual and $89.4 \%$ (101 out of 113) powered drill bone biopsies provided sufficient specimen yield and quality.

\section{Radiation Exposure of Manual and Powered Drill Bone Biopsy in Relation to Anatomical Localization}

$28 \%$ (53 out of 187) of all procedures were performed at SOMATOM AS,$+ 57 \%$ (106 out of 187) at SOMATOM Definition Flash and 15\% (28 out of 187) at SOMATOM Force. Major part of radiation indices values, all median $\mathrm{CTDI}_{\mathrm{vol}}$ values except for ilium and all median DLP values for the whole procedure including pre-bioptic scans for planning, the biopsy-guiding scans and the post-bioptic scans for documentation and exclusion of immediate complications of the biopsy were lower with powered drill biopsy (Tables 2 and 3). Similar to the radiation dose distribution of the whole procedure, the major part of median $\mathrm{CTDI}_{\mathrm{vol}}$ and DLP values as well as IQRs of the biopsy-guiding scans were lower with the powered-drill approach (Fig. 2). Statistical analysis revealed significantly lower $\mathrm{CTDI}_{\mathrm{vol}}$ for biopsy-guiding scans of powered-drill biopsies of ilium $(p<0.0001)$ and sacrum $(p=0.0232)$. Likewise, radiation exposure in terms of DLP for biopsyguiding sequences was significantly lower for both anatomical regions: ilium $(p=0.0008)$, sacrum $(p=0.0178)$. No statistical significant difference was found for other biopsy regions.

\section{Comparison Between Different Protocols on the CT Scanners}

Because of the higher tube current product on the SOMATOM Force, a subgroup analysis was performed with all manual versus powered drill biopsies on SOMATOM AS + and Flash: Median DLP of biopsy-guiding sequence was similar for manual biopsies with 60.7 $\mathrm{mGy} \cdot \mathrm{cm}$ (IQR 44.0-85.5) and for powered drill biopsies with $59.6 \mathrm{mGy} \cdot \mathrm{cm}$ (IQR 39.3-78.9) and showed no significant difference $(p=0.04383)$. In contrast, a median DLP of $218 \mathrm{mGy} \cdot \mathrm{cm}$ (IQR 172.8-311.0) was obtained for all manual biopsies on the SOMATOM Force. Manual biopsies on the SOMATOM Force required significantly higher DLP values compared to both SOMATOM AS + and Flash $(p<0.0001)$.

\section{Influence of Bone Lesion Characteristics on Radiation Exposure}

Regarding the density of the bone lesion, no significant difference of median DLP values was detected in both 
Table 2 Volume-weighted CT dose index $\left(\mathrm{CTDI}_{\mathrm{vol}}\right)$ of CTguided percutaneous bone biopsy with manual and batterypowered drill system for different anatomical regions

\begin{tabular}{|c|c|c|c|c|c|c|}
\hline \multirow[t]{2}{*}{ Anatomical location } & & \multirow[t]{2}{*}{$\mathrm{n}$} & \multicolumn{4}{|l|}{$\mathrm{CTDI}_{\mathrm{vol}}[\mathrm{mGy}]$} \\
\hline & & & 25th percentile & Median & 75th percentile & Mean $\pm \mathrm{SD}$ \\
\hline \multirow[t]{3}{*}{ Femur } & Total & 27 & 42.4 & 65.4 & 90.9 & $71.0 \pm 43.1$ \\
\hline & Manual & 13 & 41.4 & 56.9 & 128.5 & $80.9 \pm 47.7$ \\
\hline & Powered drill & 14 & 37.6 & 66.7 & 76.2 & $61.8 \pm 37.8$ \\
\hline \multirow[t]{3}{*}{ Ilium } & Total & 74 & 50.4 & 71.5 & 90.3 & $80.0 \pm 41.7$ \\
\hline & Manual & 27 & 62.1 & 83.5 & 128.5 & $101.3 \pm 49.2$ \\
\hline & Powered drill & 47 & 46.2 & 59.4 & 79.8 & $67.8 \pm 31.1$ \\
\hline \multirow[t]{3}{*}{ Sacrum } & Total & 27 & 58.4 & 71.6 & 115.5 & $92.3 \pm 59.2$ \\
\hline & Manual & 11 & 71.6 & 97.5 & 149.2 & $124.9 \pm 76.6$ \\
\hline & Powered drill & 16 & 49.1 & 63.1 & 83.7 & $69.9 \pm 29.0$ \\
\hline \multirow[t]{3}{*}{ Thoracic vertebrae } & Total & 28 & 34.1 & 48.3 & 86.6 & $64.6 \pm 49.6$ \\
\hline & Manual & 13 & 40.3 & 67.0 & 86.6 & $75.7 \pm 61.4$ \\
\hline & Powered drill & 15 & 29.9 & 39.7 & 89.0 & $54.9 \pm 35.9$ \\
\hline \multirow[t]{3}{*}{ Lumbar vertebrae } & Total & 31 & 52.0 & 70.9 & 115.0 & $91.1 \pm 68.2$ \\
\hline & Manual & 10 & 66.5 & 100.1 & 162.6 & $133.0 \pm 97.7$ \\
\hline & Powered drill & 21 & 48.0 & 62.5 & 90.0 & $71.2 \pm 37.3$ \\
\hline
\end{tabular}

Table 3 Dose-length product (DLP) of CT-guided percutaneous bone biopsy with manual and battery-powered drill system for different anatomical regions

\begin{tabular}{|c|c|c|c|c|c|c|}
\hline \multirow[t]{2}{*}{ Anatomical location } & & \multirow[t]{2}{*}{$\mathrm{n}$} & \multicolumn{4}{|l|}{$\mathrm{DLP}[\mathrm{mGy} \cdot \mathrm{cm}]$} \\
\hline & & & 25th percentile & Median & 75th percentile & Mean \pm SD \\
\hline \multirow[t]{3}{*}{ Femur } & Total & 27 & 179.3 & 332.0 & 522.0 & $366.3 \pm 212.6$ \\
\hline & Manual & 13 & 203.1 & 410.0 & 682.8 & $442.5 \pm 250.3$ \\
\hline & Powered drill & 14 & 127.7 & 302.8 & 403.1 & $295.5 \pm 146.3$ \\
\hline \multirow[t]{3}{*}{ Ilium } & Total & 74 & 327.7 & 419.8 & 529.8 & $458.9 \pm 211.1$ \\
\hline & Manual & 27 & 322.0 & 489.0 & 471.8 & $524.7 \pm 255.0$ \\
\hline & Powered drill & 47 & 328.5 & 399.7 & 695.0 & $421.0 \pm 173.2$ \\
\hline \multirow[t]{3}{*}{ Sacrum } & Total & 27 & 341.0 & 492.6 & 740.0 & $537.0 \pm 248.2$ \\
\hline & Manual & 11 & 496.0 & 626.6 & 740.0 & $659.3 \pm 287.6$ \\
\hline & Powered drill & 16 & 316.1 & 403.7 & 515.3 & $453.0 \pm 182.0$ \\
\hline \multirow[t]{3}{*}{ Thoracic vertebrae } & Total & 28 & 193.5 & 315.7 & 577.7 & $418.3 \pm 331.6$ \\
\hline & Manual & 13 & 267.0 & 392.0 & 596.0 & $473.3 \pm 316.3$ \\
\hline & Powered drill & 15 & 165.8 & 206.9 & 402.1 & $370.6 \pm 347.9$ \\
\hline \multirow[t]{3}{*}{ Lumbar vertebrae } & Total & 31 & 263.4 & 402.0 & 545.8 & $437.2 \pm 259.2$ \\
\hline & Manual & 10 & 384.8 & 520.5 & 619.0 & $559.0 \pm 279.8$ \\
\hline & Powered drill & 21 & 240.4 & 314.5 & 452.1 & $379.2 \pm 233.7$ \\
\hline
\end{tabular}

manual osteolytic $(101.0 \mathrm{mGy} \cdot \mathrm{cm}$; IQR 52.0-207.3) and osteoblastic (86.0 $\mathrm{mGy} \cdot \mathrm{cm}$; IQR 64.0-124.0) biopsies ( $p=0.6526)$. Similarly, the doses of powered drill biopsies did not differ significantly at a median DLP of 62.0 $\mathrm{mGy} \cdot \mathrm{cm}$ (IQR 44.6-81.0) for osteolytic and $66.0 \mathrm{mGy} \cdot \mathrm{cm}$ (IQR 43.4-78.9) for osteoblastic lesions $(p=0.8592)$. In contrast, the choice of biopsy system for both osteolytic $(p=0.0007)$ and osteoblastic $(p=0.0105)$ bone lesions showed significantly lower DLP values for the powered drill biopsies. In terms of depth of bone biopsy, the radiation exposure of the biopsy-guiding scans of the manual biopsies was significantly lower for the superficial biopsies with a median DLP of $53.5 \mathrm{mGy} \cdot \mathrm{cm}(\mathrm{IQR} 45.3-64.0)$ than for the deep biopsies with $88.0 \mathrm{mGy} \cdot \mathrm{cm}$ (IQR 81.0-98.0) $(p<0.0001)$. Similarly, the median DLP values of the superficial powered drill biopsies $(44.6 \mathrm{mGy} \cdot \mathrm{cm}, \mathrm{IQR}$ 29.0-67.6) were significantly lower than those of the deep biopsies (73.5 $\mathrm{mGy} \cdot \mathrm{cm}, \mathrm{IQR} 51.1-91.6)(p<0.0001)$. In addition, dose values in terms of DLP were significantly lower with powered drill compared with manual biopsy for both superficial $(p=0.0002)$ and deep $(p=0.0441)$ biopsies. The distribution of the suspected etiology of the bone lesions could be determined as follows (manual/powered drill): $21.6 \% / 15.9 \%$ primary bone tumor, $75.7 \% / 79.7 \%$ 
Fig. 2 Distribution of volumeweighted CT dose index $\left(\mathrm{CTDI}_{\mathrm{vol}}\right)$ and dose-length product (DLP) of CT-guided percutaneous bone biopsy with manual and battery-powered drill system for different anatomical locations.

Highlighting radiation exposure indices of biopsy-guiding scans with exclusion of radiation exposure of topogram, pre- and post-bioptic scans to emphasize radiation exposure differences between manual and batterypowered drill bone biopsy approaches. Whiskers represent min to max. Asterisks indicate significant difference $(p<0.05)$
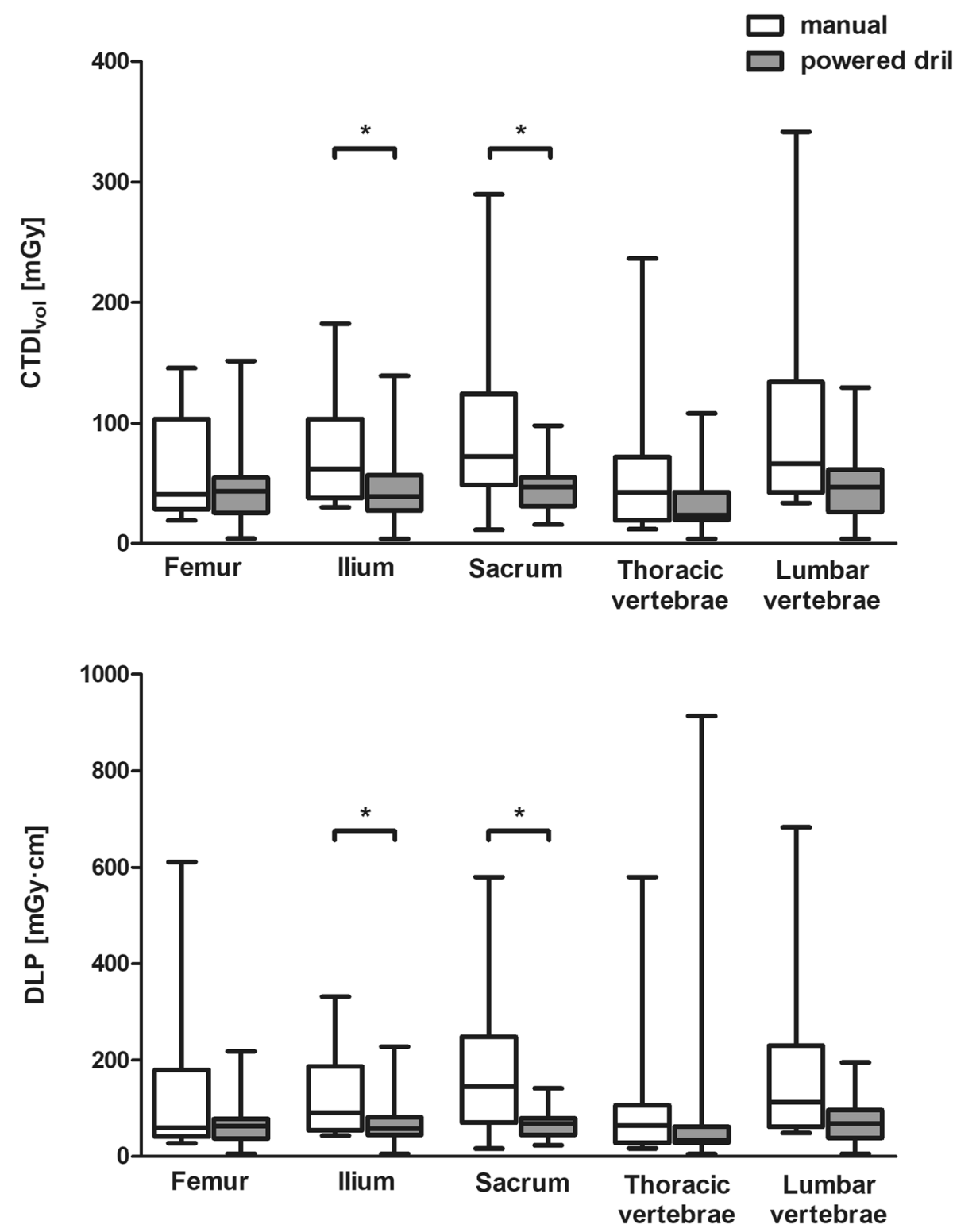

bone metastasis, $2.7 \% / 4.4 \%$ inflammatory process. There was no difference in both manual and powered drill biopsies with respect to DLP in the biopsy-guiding scans ( $p=0.903, p=0.555$ ), excluding the inflammatory bone lesions due to too small sample size. When comparing between biopsy systems, there were significantly lower DLP values when metastases were biopsied with the powered drill (median $59.8 \mathrm{mGy} \cdot \mathrm{cm}, \mathrm{IQR}$ 42.4-78.7) compared to manual biopsy (median $85.0 \mathrm{mGy} \cdot \mathrm{cm}, \mathrm{IQR}$ 50.0-202.0) ( $p=0.0002)$. In contrast, radiation exposure for biopsy of primary bone tumors did not differ significantly between the manual (median $95.5 \mathrm{mGy} \cdot \mathrm{cm}, \mathrm{IQR}$ 48.8-182.8) and powered drill (median $72.9 \mathrm{mGy} \cdot \mathrm{cm}, \mathrm{IQR}$ 37.8-98.6) system $(p=0.1379)$. All included biopsies were performed by one of two interventional radiologists with several years of experience with both biopsy systems.
In terms of an interoperator variability on radiation exposure, no trend was observed in terms of diagnostic yield or dose differences. Regarding the different needle diameters used in the manual bone biopsies of $10 \mathrm{G}, 11 \mathrm{G}$ and $13 \mathrm{G}$, Kruskal-Wallis test with Dunn-Bonferroni post hoc test showed no significant difference in the DLP values of the biopsy-guiding scans $(p=0.5599)$.

\section{Establishment of Local Diagnostic Reference Levels}

Local DRLs at our institution for CT-guided bone biopsies could be depicted as follows $\left(\mathrm{CTDI}_{\mathrm{vol}} / \mathrm{DLP}\right): \mathbf{A}: 91 \mathrm{mGy} /$ $522 \mathrm{mGy} \cdot \mathrm{cm}, \mathbf{B}: 90 \mathrm{mGy} / 530 \mathrm{mGy} \cdot \mathrm{cm}, \mathbf{C}: 116 \mathrm{mGy} / 740$ $\mathrm{mGy} \cdot \mathrm{cm}, \mathbf{D}: 87 \mathrm{mGy} / 578 \mathrm{mGy} \cdot \mathrm{cm}$ and E: $115 \mathrm{mGy} / 546$ $\mathrm{mGy} \cdot \mathrm{cm}$. 


\section{Procedural Duration of CT-guided Percutaneous Bone Biopsies}

Lowest median procedural duration of the CT-guided bone biopsy with exclusion of pre- and post-bioptic scans was depicted for ilium with 21.0 (IQR 16.6-27.7) minutes, highest for lumbar vertebrae with 23.3 (IQR 17.5-32.1) minutes (Table 4). Comparing manual and powered drill approaches, median procedural durations were between 2.2 (A) and $6.6 \mathrm{~min}$ (E) less for powered drill approaches. Nonetheless, Kruskal-Wallis test revealed no significant difference between subgroups $(p=0.512)$. No significant difference in duration was also found with respect to the density of the bone lesion $(p=0.1477)$.

\section{Discussion}

In this study, the comparison of manual and powered drill CT-guided percutaneous bone biopsies revealed significantly lower radiation exposure for biopsy-guiding scans of ilium and sacrum with a powered drill biopsy system, and radiation exposure indices were also slightly lower for the other evaluated anatomical locations. Furthermore, our study demonstrated that for both osteolytic and osteoblastic bone lesions, as well as for superficial and deep biopsies, radiation exposure was lower with the powered drill system. Hence, further dose reduction in CT-guided bone

Table 4 Duration of CT-guided percutaneous bone biopsy with manual versus battery-powered drill system at different anatomical regions

\begin{tabular}{lllll}
\hline Region & & $\mathrm{n}$ & \multicolumn{2}{c}{ Duration [min] } \\
\cline { 3 - 5 } Femur & & & Median & IQR \\
\hline \multirow{4}{*}{ Ilium } & Total & 27 & 22.7 & $16.4-27.9$ \\
& Manual & 13 & 24.7 & $18.6-40.5$ \\
& Powered drill & 14 & 22.5 & $16.3-26.3$ \\
\multirow{4}{*}{ Sacrum } & Total & 74 & 21.0 & $16.6-27.7$ \\
& Manual & 27 & 23.5 & $16.8-31.3$ \\
& Powered drill & 47 & 19.2 & $15.0-26.8$ \\
Thoracic vertebrae & Total & 27 & 21.9 & $15.7-26.4$ \\
& Manual & 11 & 25.1 & $15.7-29.2$ \\
& Powered drill & 16 & 20.6 & $15.9-24.2$ \\
Lumbar vertebrae & 28 & 22.0 & $11.5-31.7$ \\
& Manual & 13 & 23.4 & $14.4-31.7$ \\
& Powered drill & 15 & 18.7 & $9.7-32.7$ \\
& Total & 31 & 23.3 & $17.5-32.1$ \\
& Manual & 10 & 28.7 & $16.9-39.7$ \\
& Powered drill & 21 & 22.1 & $18.1-30.3$ \\
\hline
\end{tabular}

biopsies is achievable by using powered drill biopsy systems. Furthermore, a slight but not significant decrease in procedural duration could be depicted for the powered drill approaches.

Bone biopsies play a key role for several diseases causing skeletal lesions such as infectious or malignant processes [10]. CT guidance is used for biopsies of many anatomical locations within the body as it improves identification of a pathology, enables planning of access route through the body and reduces costs and interventional risks compared with open biopsy [16]. Battery-powered drill bone biopsy systems such as the commercially available Arrow ${ }^{\circledR}$ OnControl ${ }^{\circledR}$ rotatory drill pose an alternative approach to place a bioptic needle in the desired depth within a skeletal lesion opposed to the manual approach with inserting the needle by manual pressure and hammering technique $[7,17]$. However, the main objective of a CT-guided percutaneous biopsy, whether using a manual or powered drill biopsy system, is to provide a sufficient amount of biopsy material, and thus the diagnostic yield should be equivalent for both approaches as a matter of priority. In this coherence, several studies reported a sufficient diagnostic yield of both manual and powered drill CT-guided bone biopsies $[4,10]$. Our results showed that a high diagnostic yield, comparable to other studies, was given for both approaches and was slightly higher for the powered drill biopsies. Nevertheless, differences in radiation burden and procedural duration might optimize patient care and minimize radiation exposure [10]. Therefore, radiation protection aspects of CT-guided bone biopsies are worth to consider. Several studies reported dose assessments: For example, Yang et al. reported radiation exposures with a median DLP of $733 \mathrm{mGy} \cdot \mathrm{cm}$ (IQR 462-1086 $\mathrm{mGy} \cdot \mathrm{cm}$ ) [11]. Our results, like the study by Lee et al. comparing manual and powered drilling systems (mean $\pm \mathrm{SD} \mathrm{CTDI}_{\mathrm{vol}}$ : manual $270 \pm 48 \mathrm{mGy}$, powered drill $164 \pm 35 \mathrm{mGy}$ ), demonstrated that the radiation exposure was significantly lower with the powered drill approach [12]. In contrast, it also has been reported that radiation exposure was slightly higher with the power drill (DLP $1203 \mathrm{mGy} \cdot \mathrm{cm}$ ) than with the manual biopsy system (DLP $971 \mathrm{mGy} \cdot \mathrm{cm}$ ) [4].

Various factors such as the anatomical location and etiology of the bone lesion, as well as the choice of biopsy system, influence the diagnostic yield of CT-guided bone biopsies [18-21]. In this context, different features also influence radiation exposure of the biopsy procedure. For example, densely and sclerotic lesions are more difficult to be attained both with manual needle and powered drill [18, 19]. Therefore, more biopsy-guiding CT scans are likely to be required in such cases, increasing the radiation exposure and also procedural duration [10]. Kihira et al. reported radiation exposure of bone biopsies differentiated 
by density to be higher for manual than for powered drill biopsies with mean DLP values between 752 and 1317 $\mathrm{mGy} \cdot \mathrm{cm}[10]$. In our study, the results demonstrated that the density of a bone lesion, i.e., osteolytic or osteoblastic, had no significant effect on radiation exposure, but the use of the biopsy system did. With regard to an interoperator variability, our study showed no significant differences in the radiation exposures of the bone biopsies. In addition to diagnostic yield and radiation exposure, characteristics of the bone lesion and the biopsy system are also thought to influence procedural duration. Comparable to the results of Cohen et al., our study demonstrated that procedural duration of all evaluated anatomical locations was slightly lower with the powered drill biopsy system, although this time saving was not significant [4]. Although the powered drill approach is reported to offer shorter scanning time for biopsies of densely sclerotic lesions in addition to less specimen artifacts [10, 18], no significant difference in procedural duration between biopsies of osteolytic and osteoblastic lesions was observed in our study. Aside from considerations related to radiation exposure and duration, many factors influence the choice of a bone biopsy system such as availability, costs and operator preference [10]. Therefore, not only do local preferences differ with respect to biopsy systems and procedures, but also the radiation exposures of $\mathrm{CT}$ examinations can vary significantly by institution [20, 21]. Helpful benchmarks for dose monitoring are DRLs which indicate typical ionizing radiation exposure values in a country, region or an institute [22]. Although the establishment of DRLs for CT interventions is more difficult compared to diagnostic examinations due to a wide variation in location and technique, the establishment of DRLs might play a crucial role for dose optimization in interventional radiology. However, not only European and national DRLs for CT-guided bone biopsies are lacking, but also reports of locally established DRLs are rare. Therefore, our local DRLs for CT-guided bone biopsies of the most common anatomical locations might be an another step toward the establishment of national or European DRLs.

Limitations of our study are the retrospective design and that there were no equivalent numbers of manual and powered drill biopsies and partly different protocols on the CT scanners. With regard to the CT scanners used, comparison of manual biopsies on SOMATOM Force versus biopsies on SOMATOM AS + and Flash with same settings showed that DLP values were significantly higher on the SOMATOM Force. Accordingly, comparable settings on all scanners and same number of cases would be a significant optimization factor. Strengths of our study include the detailed dose assessment, which enables detailed evaluation on radiation dose of manual versus powered drill biopsy approaches. Furthermore, several anatomical locations and procedural durations were evaluated.

\section{Conclusion}

In conclusion, our study demonstrated that the use of a powered drill bone biopsy system for CT-guided percutaneous bone biopsy can reduce the radiation exposure significantly for specific anatomical locations. For both osteolytic and osteoblastic bone lesions, as well as for superficial and deep biopsies, radiation exposure was lower with the powered drill system. DRLs for CT-guided bone biopsies are needed to optimize radiation protection, and our locally determined DRLs may help as benchmarks.

Funding Open Access funding enabled and organized by Projekt DEAL. This study was not supported by any funding.

\section{Declarations}

Conflict of interest D. Bos and J. Haubold were supported as Clinician Scientists and received research grants within the University Medicine Essen Academy (UMEA) program, funded by the German Research Foundation (DFG; Grant FU356/12-1) and the Faculty of Medicine, University of Duisburg-Essen.

Consent for Publication For this type of study, consent for publication is not required.

Ethical Approval All procedures performed in studies involving human participants were in accordance with the ethical standards of the institutional and/or national research committee and with the 1964 Helsinki declaration and its later amendments or comparable ethical standards. For this type of study, formal consent is not required.

Informed Consent This study has obtained IRB approval from the Faculty of Medicine, University of Duisburg-Essen, and the need for informed consent was waived.

Open Access This article is licensed under a Creative Commons Attribution 4.0 International License, which permits use, sharing, adaptation, distribution and reproduction in any medium or format, as long as you give appropriate credit to the original author(s) and the source, provide a link to the Creative Commons licence, and indicate if changes were made. The images or other third party material in this article are included in the article's Creative Commons licence, unless indicated otherwise in a credit line to the material. If material is not included in the article's Creative Commons licence and your intended use is not permitted by statutory regulation or exceeds the permitted use, you will need to obtain permission directly from the copyright holder. To view a copy of this licence, visit http://creativecommons. org/licenses/by/4.0/.

\section{References}

1. Ahlström KH, Aström KG. CT-guided bone biopsy performed by means of a coaxial biopsy system with an eccentric drill. 
Radiology. 1993;188(2):549-52. https://doi.org/10.1148/ radiology.188.2.8327713.

2. Omura MC, Motamedi K, UyBico S, et al. Revisiting CT-guided percutaneous core needle biopsy of musculoskeletal lesions: contributors to biopsy success. AJR Am J Roentgenol. 2011;197(2):457-61. https://doi.org/10.2214/AJR.10.6145.

3. Rimondi E, Staals EL, Errani C, et al. Percutaneous CT-guided biopsy of the spine: results of 430 biopsies. Eur spine J: off publ Eur Spine Soc, Eur Spinal Deform Soc, Eur Sect Cerv Spine Res Soc. 2008;17(7):975-81. https://doi.org/10.1007/s00586-0080678-x.

4. Cohen MG, McMahon CJ, Kung JW, et al. Comparison of battery-powered and manual bone biopsy systems for core needle biopsy of sclerotic bone lesions. AJR Am J Roentgenol. 2016;206(5):W83-86. https://doi.org/10.2214/AJR.15.15067.

5. Wu JS, Goldsmith JD, Horwich PJ, et al. Bone and soft-tissue lesions: what factors affect diagnostic yield of image-guided core-needle biopsy? Radiology. 2008;248(3):962-70. https://doi. org/10.1148/radiol.2483071742.

6. Cohen SC, Gore JM. Evaluation of a powered intraosseous device for bone marrow sampling. Anticancer Res. 2008;28(6B):3843-6.

7. Swords RT, Anguita J, Higgins RA, et al. A prospective randomised study of a rotary powered device (OnControl) for bone marrow aspiration and biopsy. J Clin Pathol. 2011;64(9):809-13. https://doi.org/10.1136/jclinpath-2011-200047.

8. Deak PD, Smal Y, Kalender WA. Multisection CT protocols: sex- and age-specific conversion factors used to determine effective dose from dose-length product. Radiology. 2010;257(1):158-66. https://doi.org/10.1148/radiol.10100047.

9. Shrimpton PC, Wall BF. The Increasing Importance of X Ray Computed Tomography as a Source of Medical Exposure. Radiat Prot Dosimetry. 1995;57(1-4):413-5. https://doi.org/10.1093/ oxfordjournals.rpd.a082572.

10. Kihira S, Koo C, Lee A, et al. Reduction of radiation dose and scanning time while preserving diagnostic yield: a comparison of battery-powered and manual bone biopsy systems. AJNR Am J Neuroradiol. 2020;41(3):387-92. https://doi.org/10.3174/ajnr. A6428.

11. Yang K, Ganguli S, DeLorenzo MC, et al. Procedure-specific CT dose and utilization factors for CT-guided interventional procedures. Radiology. 2018;289(1):150-7. https://doi.org/10.1148/ radiol.2018172945.

12. Lee RKL, Ng AWH, Griffith JF. CT-guided bone biopsy with a battery-powered drill system: preliminary results. AJR Am J
Roentgenol. 2013;201(5):1093-5. https://doi.org/10.2214/ajr.12. 10521.

13. Kloeckner R, dos Santos DP, Schneider J, et al. Radiation exposure in CT-guided interventions. Eur $\mathrm{J}$ Radiol. 2013;82(12):2253-7. https://doi.org/10.1016/j.ejrad.2013.08.035.

14. European Commission. European guidelines on quality criteria for computed tomography. EUR, vol 16262. Luxembourg: Office for Official Publications of the European Communities; 1999.

15. National Council on Radiation Protection and Measurements. NCRP Report No. 172 - Reference levels and achievable doses in medical and dental imaging. Recommendations for the United States. Bethesda: National Council on Radiation Protection and Measurements; 2012.

16. Shpilberg KA, Delman BN, Tanenbaum LN, et al. Radiation dose reduction in CT-guided spine biopsies does not reduce diagnostic yield. AJNR Am J Neuroradiol. 2014;35(12):2243-7. https://doi. org/10.3174/ajnr.A4053.

17. Huh EH, Yi PH, Ray DM, et al. Comparison of powered drill \& manual bone biopsy systems: does the diagnostic yield justify the cost? J Clin Neurosci: Off J Neurosurg Soc Australas. 2020;73:125-9. https://doi.org/10.1016/j.jocn.2020.01.004.

18. Chang I-YJ, Ilaslan H, Sundaram M, et al. CT-guided percutaneous biopsy of sclerotic bone lesions: diagnostic outcomes. Skeletal Radiol. 2018;47(5):661-9. https://doi.org/10.1007/ s00256-017-2828-x.

19. Chang CY, Simeone FJ, Huang AJ. Battery-powered bone drill: caution needed in densely blastic lesions. Skeletal Radiol. 2015;44(12):1845-8. https://doi.org/10.1007/s00256-015-2241-2.

20. Smith-Bindman R, Lipson J, Marcus R, et al. Radiation dose associated with common computed tomography examinations and the associated lifetime attributable risk of cancer. Arch Intern Med. 2009;169(22):2078-86. https://doi.org/10.1001/ archinternmed.2009.427.

21. Héliou R, Normandeau L, Beaudoin G. Towards dose reduction in CT: patient radiation dose assessment for CT examinations at university health center in Canada and comparison with national diagnostic reference levels. Radiat Prot Dosimetry. 2012;148(2):202-10. https://doi.org/10.1093/rpd/ncr024.

22. Vassileva J, Rehani M. Diagnostic reference levels. AJR Am J Roentgenol. 2015;204(1):W1-3. https://doi.org/10.2214/AJR.14. 12794.

Publisher's Note Springer Nature remains neutral with regard to jurisdictional claims in published maps and institutional affiliations. 